\begin{tabular}{|c|c|c|c|}
\hline \multirow[t]{2}{*}{ TABLE 1} & \multicolumn{3}{|c|}{$\begin{array}{l}\text { Cross tabulation (intent-to-treat analysis at week } \\
\text { 24) comparing quit rates relative to physical (i.e. } \\
\text { the Fagerström Test for Nicotine Dependence } \\
\text { (FTND)) and behavioural dependence (i.e. the } \\
\text { Glover-Nilsson Smoking Behavioral } \\
\text { Questionnaire (GN-SBQ)) in the Paipo users }\end{array}$} \\
\hline & & Relapse & Quit \\
\hline \multicolumn{2}{|c|}{ High FTND and high GN-SBQ } & 5/16 (31.3) & $11 / 16(68.7)$ \\
\hline \multicolumn{2}{|c|}{ High FTND and low GN-SBQ } & $27 / 30(90.0)$ & $3 / 30(10.0)$ \\
\hline \multicolumn{2}{|c|}{ Low FTND and high GN-SBQ } & 3/8 (37.5) & $5 / 8(62.5)$ \\
\hline \multicolumn{2}{|c|}{ Low FTND and low GN-SBQ } & 5/6 (83.3) & $1 / 6(16.7)$ \\
\hline \multicolumn{2}{|l|}{ Total } & $40 / 60(66.7)$ & 20/60 (33.3) \\
\hline
\end{tabular}

directions in a smoking cessation study, with smokers losing confidence in their ability to quit experiencing a reduction in their level of motivation, and smokers achieving success boosting them up. We could not establish with confidence whether motivation levels were still balanced between groups throughout the duration of the study.

Lastly, it was suggested to expand upon the correlation between behavioural (i.e. GN-SBQ) and physical (i.e. the Fagerström Test for Nicotine Dependence (FTND)) dependence to clarify if they are independent of each other or correlated, in the assumption that a smoker highly dependent on nicotine would also tend to be more behaviourally dependent. In our study, the results of the logistic model analyses indicate that only the use of Paipo is an important predictor of success in smokers with high GN-SBQ score (OR 8.45), whereas FTND has no impact in the model. We also carried out additional analyses in the Paipo users (table 1); cross tabulation of quit rates at week 24 (intent-to-treat analysis) showed that in smokers with low GN-SBQ, chance of success was much higher in low FTND (16.7\% success rate) compared to high FTND group (only $10.0 \%$ success rate). However, the numbers in some of the cells is so small that no sound conclusion can be reached about potentially important interactions between behavioural and physical dependence.

Perhaps, as sensibly pinpointed by K. Fagerström, the main strength of this study is that it has the potential to open up a new line of research, in which improved characterisation of behavioural sub-phenotypes in smokers undergoing smoking cessation programmes can be exploited to advance current understanding of tobacco dependence and its management.

\section{P. Caponnetto*\#, F. Cibella" , A. Fisichella ${ }^{\#}$ and R. Polosa*,\#}

${ }^{*}$ Centro per la Prevenzione e Cura del Tabagismo (CPCT), AOU "Policlinico-Vittorio Emanuele", Università di Catania, "Institute of Internal Medicine, AOU "Policlinico-Vittorio Emanuele", Università di Catania, Catania, and "Istituto di Biomedicina e Immunologia Molecolare del Consiglio Nazionale delle Ricerche, Palermo, Italy.

Correspondence: R. Polosa, UOC di Medicina Interna, Edificio 4, Piano 3, AOU "Policlinico-Vittorio Emanuele", Università di Catania, Via S. Sofia 78, 95123 Catania, Italy. E-mail: polosa@unict.it

Statement of Interest: None declared.

\section{REFERENCES}

1 Caponnetto P, Cibella F, Mancuso S, et al. Effect of a nicotine-free inhalator as part of a smoking-cessation programme. Eur Respir J 2011; 38: 1005-1011.

2 Glover ED, Nilsson F, Westin A, et al. Developmental history of the Glover-Nilsson Smoking Behavioral Questionnaire. Am J Health Behav 2005; 29: 443-455.

3 Caponnetto P, Polosa R. Common predictors of smoking cessation in clinical practice. Respir Med 2008; 102: 1182-1192.

4 Caponnetto P, Polosa R. Towards an improved understanding of smoking relapse predictors: recipe for success? Addiction 2011; 6: 2122-2123.

5 Chassin L, Presson CC, Sherman SJ, et al. Long-term psychological sequelae of smoking cessation and relapse. Health Psychol 2002; 21: 438-443.

DOI: $10.1183 / 09031936.00217611$

\title{
Upper age limit for bronchiolitis: 12 months or 6 months?
}

\section{To the Editors:}

Midulla et al. [1] published their interesting results on virusspecific 1-yr outcome of infants with bronchiolitis requiring hospital treatment. Originally, 313 bronchiolitis patients aged $<12$ months with no previous wheezing histories were recruited during a 5-yr period, excluding summer times. On admission, 195 viruses were detected in 174 (55.6\%) infants by PCR from nasal wash samples. As the authors concluded, the virus detection rate was rather low [1]. When nasopharyngeal aspirates are studied and the available PCR panel covers all notable respiratory viruses, the rate can be nearly $100 \%$, as was seen in our recent study in <6-month-old infants with bronchiolitis [2].

12 months later, $262(83.7 \%)$ parents were contacted by telephone and interviewed using structured questionnaires, 
and recurrent post-bronchiolitis wheezing, defined as at least two episodes, was reported in 138 (52.7\%) cases versus four $(10.3 \%)$ in controls. Although the difference was highly significant statistically, the estimate in controls must be rough, since only 39 controls (infants hospitalised for an acute disease unrelated to the respiratory system) were enrolled. The results in controlled studies are highly dependent on how the controls have been selected and collected; for example, from the general population or from hospital patients, and with infants at risk included or excluded.

When children with and without recurrent post-bronchiolitis wheezing were compared using logistic regression as the multivariate model, rhinovirus infection (OR 3.3), family history of asthma (OR 2.6) and absence of infiltration in chest radiograph (OR 2.5) were independently significant risk factors for recurrent wheezing. Blood eosinophil count $>0.4 \times 10^{9}$ cells per $\mathrm{L}$ was not significant, though eosinophils were significant as both continuous and categorised variables in univariate analyses. Since the absence of radiological infiltration was a risk factor, the severity of infection may have less impact, and the host factors and the properties of causative viruses may have more impact on outcome.

The age distributions of different viruses were not presented [1]. In the earlier paper from the same authors covering the first three study years, the mean \pm SD age was $2.0 \pm 1.8$ months in the 60 sole respiratory syncytial virus (RSV), $3.1 \pm 2.4$ months in the 16 sole rhinovirus and $3.5 \pm 3.1$ months in the 15 RSV- and bocaviruspositive cases [3]. In the Finnish studies, RSV has been the major cause of bronchiolitis at age $<6$ months, and both RSV and rhinoviruses have caused bronchiolitis at age 6-12 months [4]. Thereafter, rhinoviruses have been the predominant wheezinginducing viruses. Compared with these previous observations, the mean age of the sole rhinovirus cases was rather low [3], highlighting the role rhinoviruses also have in young infants aged $<6$ months, or even in those aged $<3$ months. In our recent study on bronchiolitis at $<6$ months of age, RSV caused $70.5 \%$ and rhinovirus $12.7 \%$ of the 168 cases [2]. The proportion of bocavirus was $<5 \%$, and all cases were mixed infections with other viruses. Post-bronchiolitis wheezing was present in $34.6 \%$ and repeated post-bronchiolitis wheezing (at least two episodes) in $18.1 \%$ of those 127 children, who were prospectively followed up using diaries filled in by parents and/or doctors on duty until the age of 1.5 yrs [5]. These figures in $<6$-month-old bronchiolitis patients are lower than the $52.7 \%$ proportion of recurrent wheezing in the Italian children admitted at $<12$ months of age [1]. Thus, the risk of post-bronchiolitis wheezing is associated with age and causative virus on admission, which interact with each other.

There is some preliminary evidence on differences in clinical severity (lower oxygen saturations in RSV-positive cases), air trapping or patchy infiltrates in chest radiographs (RSV-positive cases) and association with atopy and eosinophilia (rhinoviruspositive cases) in relation to viral aetiology $[1,4]$. In line with this, RSV-associated and rhinovirus-associated bronchiolitis/wheezing could not be separated by clinical characteristics, but patients with RSV infection had lower oxygen saturations and patients with rhinovirus infection more often had atopy and eosinophilia in our previous study [4].
The upper age limit of bronchiolitis cannot be 24 months and that limit should be 12 months [1], or even lower [2,5]. The children who wheeze at 1-2 yrs of age form a heterogeneous group of patients with different wheezing phenotypes [4]. The research on the presence and impact of genotypes is so far at an early stage. The results of the study of MidulLA et al. [1], in line with the results of the previous and recent Finnish studies, call for clinical studies including only patients aged less than 12 months on admission. Genetic properties, clinical characteristics, viral identifications and subsequent outcomes should be incorporated in the same model, and the analyses should be carried out and adjusted for age and sex.

Currently available evidence suggest that RSV and rhinovirus at least should be studied in children hospitalised for bronchiolitis or wheezing at $<12$ months of age. The identification of viruses in wheezing infants, evaluated in relation to the age of the patient, may be more important than, for example, "allergy studies", which seem to be "à la mode" in paediatric clinical practice.

\section{Korppi, P. Koponen and K. Nuolivirta}

Pediatric Research Center, Tampere University and University Hospital, Tampere, Finland.

Correspondence: M. Korppi, Pediatric Research Center, Tampere University and University Hospital, FM-3 Building, 33014 Tampere Unversity, Tampere, Finland. E-mail: matti. korppi@uta.fi

\section{Statement of Interest: None declared.}

\section{REFERENCES}

1 Midulla F, Pierangeli A, Cangiano G, et al. Rhinovirus bronchiolitis and recurrent wheezing: 1-year follow-up. Eur Respir J 2012; 39: 396-402.

2 Koponen P, Helminen M, Paassilta M, et al. Preschool asthma after bronchiolitis in infancy. Eur Respir J 2012; 39: 76-80.

3 Midulla F, Scagnolari C, Bonci E, et al. Respiratory syncytial virus, human bocavirus and rhinovirus bronchiolitis in infants. Arch Dis Child 2010; 95: 35-41.

4 Korppi M, Kotaniemi-Syrjänen A, Waris M, et al. Rhinovirusassociated wheezing in infancy: comparison with respiratory syncytial virus bronchiolitis. Pediatr Infect Dis J 2004; 23: 995-999.

5 Nuolivirta K, Koponen P, Helminen M, et al. Weight gain in infancy and post-bronchiolitis wheezing. Acta Paediatr 2012; 101: $38-42$.

DOI: 10.1183/09031936.00170511

\section{From the authors:}

We thank M. Korppi and co-workers for their correspondence regarding our article [1]. In our prospective study, we identified rhinovirus (RV) infection and a positive family history for asthma as substantial risk factors for recurrent wheezing during 1-yr follow-up after the initial episode of bronchiolitis. The distinctive point of our study was that all the infants enrolled had clinically well-characterised bronchiolitis, defined as the first episode of acute lower airway infection that appears with a history of upper respiratory tract infection followed by acute 